\title{
Thermalization in closed quantum systems: Semiclassical approach
}

\author{
J. G. Cosme ${ }^{1}$ and O. Fialko ${ }^{2}$ \\ ${ }^{1}$ Institute for Advanced Study and Centre for Theoretical Chemistry and Physics, Massey University, Auckland, New Zealand \\ ${ }^{2}$ Institute of Natural and Mathematical Sciences and Centre for Theoretical \\ Chemistry and Physics, Massey University, Auckland, New Zealand
}

(Dated: April 27, 2022)

\begin{abstract}
Thermalization in closed quantum systems can be understood either by means of the eigenstate thermalization hypothesis or the concept of canonical typicality. Both concepts are based on quantum mechanical formalism such as spectral properties of the eigenstates or entanglement between subsystems respectively. Here we study instead the onset of thermalization of Bose particles in a two-band double well potential using the truncated Wigner approximation. This allows us to use the familiar classical formalism to understand quantum thermalization in this system. In particular, we demonstrate that sampling of an initial quantum state mimics a statistical mechanical ensemble, while subsequent chaotic classical evolution turns the initial quantum state into the thermal state.
\end{abstract}

\section{INTRODUCTION}

Thermalization is regarded as one of the most fundamental facts in physics. Its foundations and basic postulates are still the subject of debates. Recent advances in addressing this issue have brought us two quantum concepts that shed light on the onset of thermal equilibrium inside closed quantum systems. One of them is the so-called eigenstate thermalization hypothesis (ETH, $[1,2])$. ETH conjectures that under certain initial conditions the expectation values of observables of a quantum system behave as if they were thermal during later times of its evolution. In another approach, so-called canonical typicality $(\mathrm{CT},[3])$, the process of thermalization is explained via entanglement between subsystems [4]. However, the quantum ideas can be hard to imagine. Semiclassical ideas, on the other hand, may provide intuitive physical insights into quantum mechanics [5]. Here we study the onset of thermalization in a quantum system using the semiclassical truncated Wigner approximation. This allows us to use the familiar classical formalism to explain how quantum fluctuations in an initial state turn into thermal fluctuations at later times during chaotic classical evolution of Wigner trajectories.

We introduce notations to be used throughout the rest of the paper by reviewing briefly recent progress on quantum thermalization. ETH was tested against another hypothesis numerically in [6] (see also [7]). To understand ETH, consider the initial state $\left|\phi_{0}\right\rangle=\sum_{k} \alpha_{k}|k\rangle$, where $|k\rangle$ are the eigenstates of a Hamiltonian $\hat{H}$ with eigenvalues $E_{k}$. The eigenstates are thermal, which is reflected in the spectral properties as follows. The state evolves as $|\phi(t)\rangle=e^{-i \hat{H} t / \hbar}\left|\phi_{0}\right\rangle$. As a prerequisite for thermalization, the expectation value of an observable $\langle\hat{\mathcal{O}}\rangle=\sum_{k, l} \alpha_{l}^{*} \alpha_{k} e^{i\left(E_{l}-E_{k}\right) t / \hbar} \mathcal{O}_{l k}$ with $\mathcal{O}_{l k}=\langle l|\hat{\mathcal{O}}| k\rangle$, after sufficient time, must relax to the long-time average $\overline{\langle\hat{\mathcal{O}}\rangle}=\sum_{k}\left|\alpha_{k}\right|^{2} \mathcal{O}_{k k}$. ETH then states that this occurs if $\mathcal{O}_{k k}$ is a smooth function of $E_{k}$, while the off-diagonal elements $\mathcal{O}_{k l}$ are negligible. Moreover, the energy $E_{0}=\sum_{k}\left|\alpha_{k}\right|^{2} E_{k}$ must have small uncertainty $\Delta E_{0}=\sum_{k}\left|\alpha_{k}\right|^{2}\left(E_{k}-E_{0}\right)^{2}[2,[6]$ to ensure that the re- laxed state is thermal and depends only on the energy.

$\mathrm{CT}$, on the other hand, replaces the need for any ensemble averaging, the main postulate of statistical mechanics considered to be artificial. Here, thermalization is reached on the level of a subsystem. The reduced density matrix of the subsystem is canonical for the majority of the possible pure states of the entire many-body system under global constraint such as energy. This was established under great generality by invoking Levy's lemma [4]. Then, the properties of the subsystem can be calculated using the reduced density matrix constructed from the pure density matrix of the entire system $\hat{\rho}_{p}=|\phi(t)\rangle\langle\phi(t)|$ giving the same prediction as if the entire system was in the microcanonical state $\hat{\rho}_{m}=\mathcal{N}_{E_{0}, \delta}^{-1} \sum_{k}|k\rangle\langle k|$. The sum is over $\mathcal{N}_{E_{0}, \delta}$ eigenstates $|k\rangle$, with energies lying within some window $\left[E_{0}-\delta, E_{0}+\delta\right]$ such that $\delta \ll E_{0}$.

In classical physics, thermalization is explained by means of classical chaos. Being chaotic, the system wanders all over the constant energy surface in the phase space, becoming ergodic. Averaged properties of the system over a long time can then be estimated by averaging over the accessible phase space [8]. To relate that to thermalization in closed quantum systems, it was originally argued that ETH can be explained if the quantum system is chaotic in the classical limit [2]. However, many quantum systems do not have classical counterparts; nevertheless, they thermalize [6, 9, 10]. Thermalization in such systems is believed to be related to the onset of chaotic eigenstates in the system [11, 12]. To demonstrate this. we write the density matrix corresponding to $|\phi(t)\rangle$ as

$$
\hat{\rho}_{p}=\sum_{k}\left|\alpha_{k}\right|^{2}|k\rangle\left\langle k\left|+\sum_{k \neq l} \alpha_{k} \alpha_{l}^{*} e^{-i\left(E_{k}-E_{l}\right) t / \hbar}\right| k\right\rangle\langle l| .
$$

Here the coefficients $\alpha_{k}$ are assumed to be random and independent, implying that the second term quickly averages to zero at long times. In the remaining first term, the factor $\left|\alpha_{k}\right|^{2}$ is assumed to be a smooth function with narrow width $\Delta E_{0} \ll E_{0}$, yielding $\hat{\rho}_{p} \approx \hat{\rho}_{m}$. 


\section{THE MODEL}

We study thermalization in a quantum system which is amenable to the semiclassical analysis. Bosons are trapped in a double-well potential shown in Fig. 1, They occupy four energy levels (we will refer to them also as modes) and are described by the following two-band Bose-Hubbard Hamiltonian [13]:

$$
\begin{aligned}
\hat{H} & =-\sum_{r \neq r^{\prime}, l} J^{l} \hat{b}_{r}^{l \dagger} \hat{b}_{r^{\prime}}^{l}+\sum_{r, l} U^{l} \hat{n}_{r}^{l}\left(\hat{n}_{r}^{l}-1\right)+\sum_{r, l} E_{r}^{l} \hat{n}_{r}^{l} \\
& +U^{01} \sum_{r, l \neq l^{\prime}}\left(2 \hat{n}_{r}^{l} \hat{n}_{r}^{l^{\prime}}+\hat{b}_{r}^{l \dagger} \hat{b}_{r}^{l \dagger} \hat{b}_{r}^{l^{\prime}} \hat{b}_{r}^{l^{\prime}}\right)
\end{aligned}
$$

where $\hat{b}_{r}^{l \dagger}$ and $\hat{b}_{r}^{l}$ are the bosonic creation and annihilation operators respectively of an atom in well $r$ and energy level $l$. The parameters in the Hamiltonian can be easily evaluated for a specific double-well potential [13]. The ground and first excited-state energies are $E_{r}^{l}=\int d x \phi_{r}^{l *}(x) \hat{H}_{s p} \phi_{r}^{l}(x)$. The tunneling term between wells is $J^{l}=-\int d x \phi_{L}^{l *}(x) \hat{H}_{s p} \phi_{R}^{l}(x)$. The interaction term between atoms in the same well and on the same energy level is $U^{l}=g \int d x\left|\phi_{r}^{l}\right|^{4}$ and on different energy levels is $U^{01}=g \int d x\left|\phi_{r}^{0}(x)\right|^{2}\left|\phi_{r}^{1}(x)\right|^{2}$. This term contributes to atoms changing energy levels. We consider a harmonic potential with oscillator frequency $\omega_{0}$, which is split by a focused laser beam located at the center of the trap and described by a Gaussian potential $V_{0} \exp \left(-x^{2} / 2 \sigma^{2}\right)$. The barrier height is chosen to be $V_{0}=5 \hbar \omega_{0}$ with width $\sigma=0.1 l_{h o}$, where the harmonic oscillator length is $l_{h o}=\sqrt{\hbar / m \omega_{0}}$. The localized functions $\phi_{r}^{l}$ are obtained by numerically solving the eigenstates of the single particle Hamiltonian $\hat{H}_{s p}$. The coupling $g$ can be varied by Feshbach resonance in an experiment. Parameters in units of the harmonic confinement $\hbar \omega_{0}$ are $J^{0}=0.26, J^{1}=0.34, E_{r}^{0}=1.25, E_{r}^{1}=3.17, U^{0}=4 / N$, $U^{1}=3 U^{0} / 4$, and $U^{01}=U^{0} / 2$. For $N=40$ particles, the system is complex enough to show thermalization and

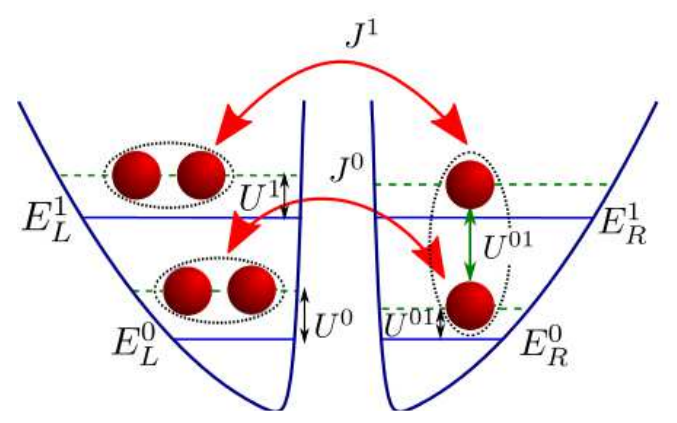

FIG. 1. Schematic of the double-well potential with two energy bands. The diagram shows the tunneling of particles and how the energy levels change due to the interactions between them. The interband coupling $U^{01}$ makes the system complex enough to show quantum thermalization of the particles. can be studied by exact diagonalization. A small number of modes and large number of particles allow us to study the system also in the semiclassical limit.

\section{TRUNCATED WIGNER APPROXIMATION}

For large $N$, we can use the semiclassical truncated Wigner approximation (TWA). The leading corrections due to finiteness of $N$ is of the order $1 / N^{2}[14]$. For $N=40$ we can safely ignore such terms and the results can be compared to exact diagonalization (see below). The use of TWA is justified since the initial state sampling naturally mimics the ensemble averaging in statistical mechanics. As a matter of fact, it was conjectured that it is probable that each Wigner trajectory approximately corresponds to a single realization of experiments [14]. Within TWA the operators are treated as complex numbers, $b_{r}^{l}$ and $b_{r}^{l *}$, satisfying the following set of nonlinear equations:

$$
i \hbar \frac{\partial b_{r}^{l}}{\partial t}=\frac{\partial H_{W}}{\partial b_{r}^{l *}}
$$

where $H_{W}=\langle\beta|\hat{H}| \beta\rangle$ is the Weyl-ordered Hamiltonian operator. It is calculated by replacing $\hat{b}_{r}^{l} \rightarrow\left(b_{r}^{l}+\frac{1}{2} \frac{\partial}{\partial b_{r}^{l *}}\right)$ and $\hat{b}_{r}^{l \dagger} \rightarrow\left(b_{r}^{l *}-\frac{1}{2} \frac{\partial}{\partial b_{r}^{l}}\right)$ in Eq. (2) [14]. We solve Eq. (3) by sampling appropriately an initial quantum state. If the initial state is a Fock state, $b_{r}^{l}$ are sampled for large $N$ with fixed amplitude $\sqrt{n_{r}^{l}}$ and uniformly random phase $\theta$, i.e., $b_{r}^{l}=\sqrt{n_{r}^{l}} \exp (i \theta)$ [15]. We also study dynamics from the coherent state sampled as $b_{r}^{l}=\sqrt{n_{r}^{l}}+1 / 2\left(\eta_{1}+i \eta_{2}\right)$, where $\eta_{j}$ are real normal Gaussians. These have the correlations $\overline{\eta_{j}}=0$ and $\overline{\eta_{j} \eta_{k}}=\delta_{j k}$, where the overline denotes an average over many samples [15]. The occupation numbers can be calculated by averaging $\left|b_{r}^{l}(t)\right|^{2}$ over many realizations from the initial sampling. To ensure convergence the number of trajectories and number of particles are taken to be large, $N=10^{4}$.

The dynamics of ultracold atoms in a single-band double well potential has been studied extensively [16]. This system can be mapped onto the classical pendulum with small Josephson oscillations and the self-trapping regime of atoms being identified with small-amplitude oscillations and full rotation of the pendulum around its pivot point, respectively. Similarly, a two-band double-well potential filled with cold atoms can be described as two nontrivially coupled nonrigid pendulums in the semiclassical limit 17]. As a result, there are regimes, where the system is chaotic [18]. With our choice of parameters, the Wigner trajectories are indeed chaotic as shown below.

Thermal equilibration through chaotic semiclassical dynamics has been studied before, e.g., in the Dicke model [19] and in trapped atomic gases with spin-orbit coupling [20]. In this paper we extend that by showing in detail how an initial quantum state turns into the thermal 


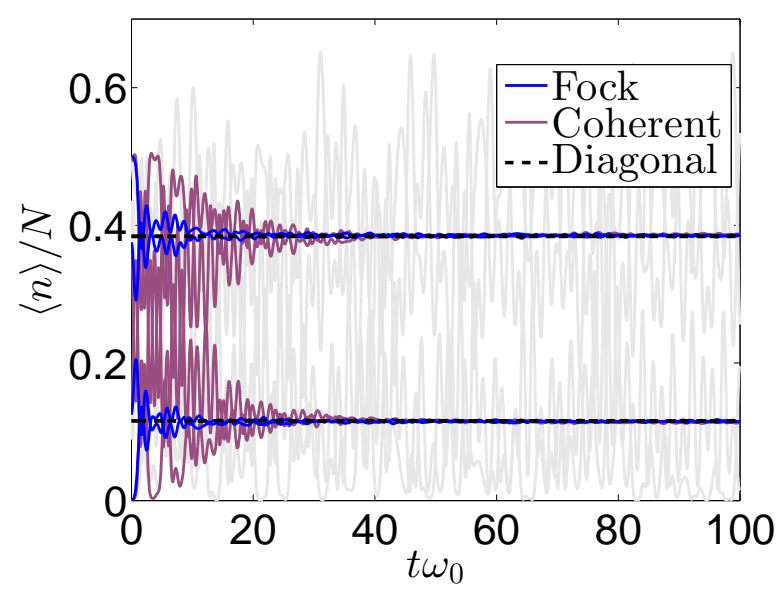

FIG. 2. TWA dynamics of the occupation numbers in each mode from Fock and coherent initial states with the same energy $E_{0} / N \approx 2.25 \hbar \omega_{0}$ and narrow energy variances $\Delta E_{0} / E_{0}=0.09$ and 0.02 respectively. They equilibrate at values which are in excellent agreement with the quantum diagonal ensembles prediction for $N=40$. A single TWA trajectory is shown in semitransparent gray; it exhibits chaotic behavior. Only averaging over many such trajectories leads to the correct relaxed values of the occupation numbers.

state through chaotic evolution. The proper sampling of an initial quantum state mimics a statistical mechanical ensemble, while ergodicity of Wigner trajectories drives the initial pure distribution to the thermal one.

\section{EXACT DIAGONALIZATION}

The semiclassical analysis will be compared with the predictions of the full quantum dynamics for consistency. We use the Fock basis $|n\rangle=\left|n_{L}^{0}\right\rangle \otimes\left|n_{R}^{0}\right\rangle \otimes\left|n_{L}^{1}\right\rangle \otimes\left|n_{R}^{1}\right\rangle$, where $\left|n_{r}^{l}\right\rangle$ is a number state on level $l$ and site $r$. The eigenstates are $|k\rangle=\sum_{n} C_{n}^{k}|n\rangle$, where $C_{n}^{k}$ are extracted from exact diagonalization of the Hamiltonian (2) for $N=40$ particles. Assume that the initial state $\left|\phi_{0}\right\rangle=\left|n_{0}\right\rangle$ is a Fock state with fixed energy $E_{0}$, implying that the coefficients $\alpha_{k} \equiv C_{n_{0}}^{k *}$ are obtained from the exact diagonalization (ED). The details of the initial state are irrelevant for subsequent evolution if its energy variance is small, as we discussed above. The system for any such initial state with the same energy $E_{0}$ relaxes to the diagonal ensemble $\hat{\rho}_{m}=\sum_{k}\left|C_{n_{0}}^{k}\right|^{2}|k\rangle\langle k|$.

Another commonly used state albeit quite different from the Fock state, is a coherent state, which is a superposition of all possible Fock states, $|\beta\rangle=$ $e^{-|b|^{2} / 2} \sum_{n=0}^{\infty}\left(b^{n} / \sqrt{n !}\right)|n\rangle$. Inserting the resolution of identity expressed via the Fock basis, we get $\langle\beta|\hat{H}| \beta\rangle=$ $\sum_{n, m}\langle\beta \mid n\rangle\langle m \mid \beta\rangle E_{n m}$, where $E_{n m}=\langle n|\hat{H}| m\rangle$. We split it into the sums over diagonal and off-diagonal terms, $\langle\beta|\hat{H}| \beta\rangle=\sum_{n} e^{-|b|^{2}} \frac{|b|^{2 n}}{n !} E_{n}+\sum_{n \neq m} e^{-|b|^{2}} \frac{b^{* n} b^{m}}{\sqrt{n ! m !}} E_{n m}$. Since we require the energy variance in the Fock basis
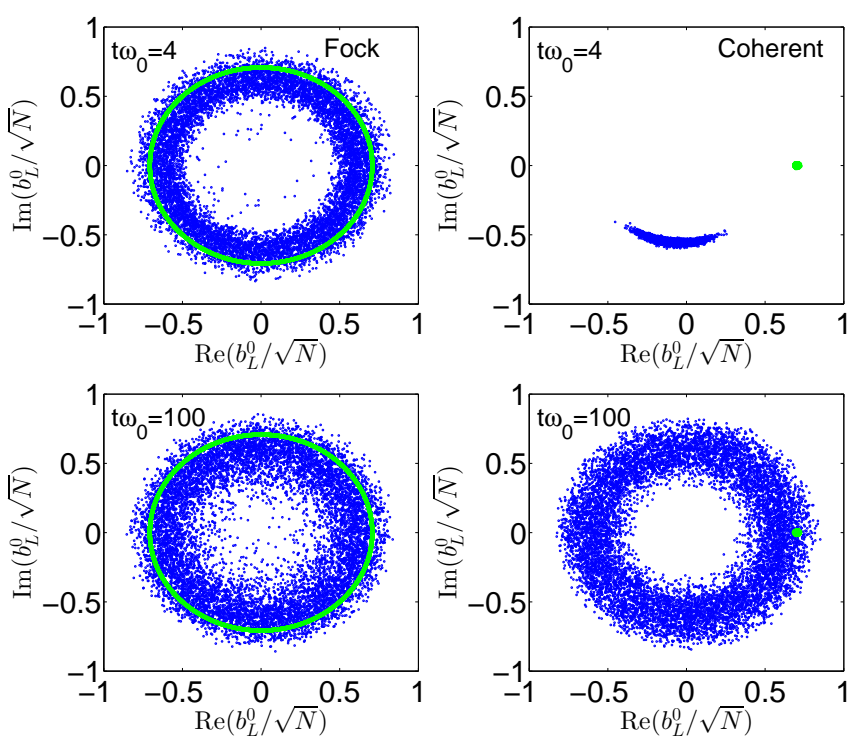

FIG. 3. Ergodicity in TWA. As an example we show Wigner distributions of one of the lower levels. Initially the system is prepared in the Fock state (green cycle shown on the left) or in the coherent state (green bump shown on the right). Trajectories sampled from the initial states fill the available phase space as time evolves (blue dots). The distributions become essentially the same at some time and after that they do not change, which suggests thermalization is reached within TWA. In this sense, quantum fluctuations of the initial state turn into thermal fluctuations in the course of evolution.

to be small, which translates to $\sqrt{\sum_{m \neq n} E_{n m}^{2}} \ll E_{n}$, the off-diagonal elements in the Hamiltonian matrix $E_{n m}$ are negligibly small as compared to the diagonal elements $E_{n}$. Therefore we neglect the terms with $n \neq m$. For a quantum system with large number of particles in each mode, $|b|^{2} \gg 1$, the Poissonian factor $e^{-|b|^{2}} \frac{|b|^{2 n}}{n !}$ becomes sharply peaked at around $n=|b|^{2}$. This allows us to approximate $\langle\beta|\hat{H}| \beta\rangle \approx\langle n|\hat{H}| n\rangle$ with $n=|b|^{2}$. The energy variance of the coherent state can be shown to get progressively small when we increase the number of particles. Therefore, it is supposed to relax to the diagonal ensemble $\hat{\rho}_{m}$ if it has the same energy $E_{0}$. We examine this within the semiclassical approach.

\section{RESULTS}

To compare ED and TWA, we scale all quantities with $N$. We study the dynamics from initial Fock and coherent states with the same energy $E_{0} / N \approx 2.25 \hbar \omega_{0}$. The relaxation dynamics from TWA calculations is shown in Fig. 2. The final relaxed values of the occupation numbers are in excellent agreement with the quantum diagonal ensembles prediction. For the two different initial conditions the upper and lower modes thermalize at $n_{L}^{0} \approx n_{R}^{0} \approx 0.4 N, n_{L}^{1} \approx n_{R}^{1} \approx 0.1 N$. The independence on initial conditions is the hallmark of thermaliza- 
tion. The Wigner trajectories exhibit chaotic behavior by quickly filling the available phase space, as shown in Fig. 3. This is quite similar to classical thermalization, where such behavior of classical trajectories is the source of ergodicity in an ensemble of identical systems [8]. The quantum-mechanical fuzziness is the key to understand ergodicity in the semiclassical language: averaging over the initial sampling replaces ensemble averaging.

Within the realm of ED the diagonal ensemble $\hat{\rho}_{m}$ contains all necessary information about the relaxed state. Our aim is to calculate the population distribution in a mode $P_{n}$ and compare it with the analogous distribution derived from the TWA approximation. This quantity simply gives the occupancy of that mode, $\langle\hat{n}\rangle=\sum_{n^{\prime}} n^{\prime} P_{n^{\prime}}$, and the reduced density matrix of that mode, $\sum_{n^{\prime}} P_{n^{\prime}}\left|n^{\prime}\right\rangle\left\langle n^{\prime}\right|$. To calculate this density distribution we express $\hat{\rho}_{m}$ via the Fock basis $\hat{\rho}_{m} \approx \sum_{k, n}\left|C_{n_{0}}^{k}\right|^{2}\left|C_{n}^{k}\right|^{2}|n\rangle\langle n|$, where again we rely on the chaoticity of the eigenstates, leaving only the diagonal contribution, yielding $P_{n}=\sum_{k}\left|C_{n_{0}}^{k}\right|^{2}\left|C_{n}^{k}\right|^{2}$. We found a very good agreement with the analogous distribution obtained from the TWA calculations, as it is shown in Fig. 4. The later is obtained by noting that $n_{r}^{l} \rightarrow\left|b_{r}^{l}\right|^{2}$.

The resulting population distributions can be inferred from the ergodicity of the Wigner trajectories originating from the initial sampling which is narrow in energy. The Wigner distribution function of the entire system can thus be represented as some sharply peaked function $\bar{\delta}\left(H_{W}-E_{0}\right)$, the microcanonical analog of the quantum case. We approximate it with the Gaussian $\bar{\delta}(x)=\frac{1}{\sigma \sqrt{2 \pi}} \exp \left(-x^{2} / 2 \sigma^{2}\right)$ with $\sigma \sim \Delta E_{0}$. The Wigner distribution of a mode $b_{r}^{l}$ is then given by integrating out over all modes but one, $W_{m}\left(b_{r}^{l}, b_{r}^{l *}\right)=\int \bar{\delta}\left(H_{W}-\right.$ $\left.E_{0}\right) \prod_{l^{\prime} \neq l, r^{\prime} \neq r} d b_{r^{\prime}}^{l^{*}} d b_{r^{\prime}}^{l^{\prime}}$, which is numerically evaluated using the Monte Carlo integration. We found good agreement with the exact Wigner distributions as shown in Fig. 4. This is conceptually analogous to CT, although the underlying basis of our approach is quite distinct.

Having observed equilibration in each of the four modes, we now examine the states of each mode in more detail. Our aim is to demonstrate that the equilibrium states are thermal. In studying thermalization in closed quantum systems, the Hamiltonian of a system is usually split into several parts, $\hat{H}=\hat{H}_{S}+\hat{H}_{B}+\hat{H}_{\text {int }}$, representing the subsystem, the bath, and interactions between them leading to their mutual thermalization. Equation (2) represents such a situation: Each of the four modes can be regarded as a subsystem $\left(=\hat{H}_{S}\right)$ coupled to the rest of the system $\left(=\hat{H}_{B}\right)$, via the tunneling and coupling terms $\left(=\hat{H}_{\text {int }}\right)$. The modes may exchange energy and particles via tunneling; therefore thermal states of each mode are expected to be described by grand-canonical ensembles $\hat{\rho}_{G C} \sim e^{-\beta\left(\hat{H}_{S}-\mu \hat{n}_{S}\right)}$, where for a given mode $\hat{H}_{S}=U^{l} \hat{n}_{r}^{l}\left(\hat{n}_{r}^{l}-1\right)+E_{r}^{l} \hat{n}_{r}^{l}$ and $\hat{n}_{S}=\hat{n}_{r}^{l}$. To ensure that the resulting distributions are thermal, we fitted them with the grand-canonical distribution $\hat{\rho}_{G C}$. We have extracted $\beta^{-1} \approx 6 \hbar \omega_{0}, \mu \approx 3 \hbar \omega_{0}$ and $\beta^{-1} \approx 12 \hbar \omega_{0}$,
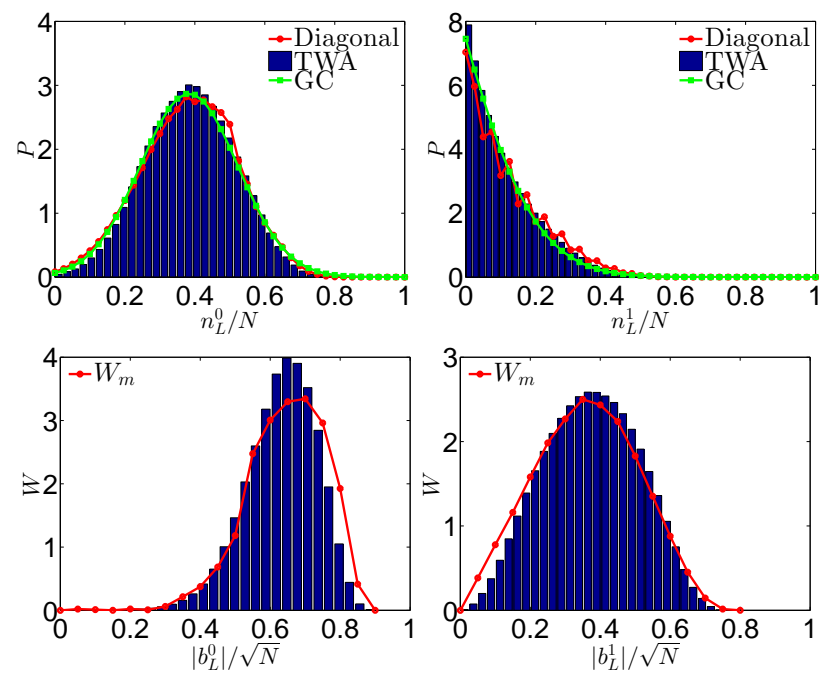

FIG. 4. (Upper panels) Comparison of the distributions $P_{n}$ derived from the diagonal ensemble with the corresponding distributions derived from the grand-canonical ensembles. Both are compared with the corresponding data extracted from TWA. (Lower panels) The good agreement between the exact Wigner distribution and the distribution $W_{m}$.

$\mu \approx 0.25 \hbar \omega_{0}$ for the lower and upper modes, respectively. In passing, we note that if we chose, e.g., the inter-band coupling $U^{01}$ to be much smaller than the current one, the situation is different: the equilibration is lost along with the chaotic behavior of the Wigner trajectories.

\section{DISCUSSIONS AND CONCLUSIONS}

We have demonstrated that each of the four modes thermalizes with the rest of the system. We did this as follows. On the one hand, each mode is weakly coupled to each other via tunneling and interaction terms in the Hamiltonian so that they can exchange particles and energy. As a result, the corresponding distributions are expected (and were shown) to be well described by the grand-canonical ensembles with appropriate temperatures and chemical potentials. On the other hand, the initial state independence of the final states provides further evidence of thermalization of each mode. Moreover, the final states of each mode can be inferred from the microcanonical ensemble of the whole system.

We have also shown that the semiclassical truncated Wigner approach and full quantum description agree in reproducing the states of each mode after they have been thermalized. While the quantum description of thermalization has been elucidated extensively in the literature [1-4, 7], in this work we analyzed the semiclassical approach in order to seek further insights into the physics of quantum thermalization. The truncated Wigner approach has revealed deep connection between thermalization in closed quantum systems and classical thermalization. Although the main postulate of classical sta- 
tistical mechanics is considered to be artificial and was replaced in quantum formalism [4], we have shown that quantum-mechanical fuzziness of initial states within the semiclassical formalism naturally supports the concept of statistical mechanical ensembles. Moreover, the ergodicity of Wigner trajectories leads to thermal relaxation. Being conceptually different, our study adds to the understanding of quantum thermalization and to the recent advances in pushing the limits of quantum thermalization and its understanding in the macroscopic limit [10, 19
21].

\section{ACKNOWLEDGMENTS}

We thank Anatoli Polkovnikov and Joachim Brand for useful and insightful comments. O.F. was supported by the Marsden Fund (Project No. MAU1205), administrated by the Royal Society of New Zealand.
[1] J. M. Deutsch, Phys. Rev. A 43, 2046 (1991).

[2] M. Srednicki, Phys. Rev. E 50, 888 (1994).

[3] H. Tasaki, Phys. Rev. Lett. 80, 1373 (1998) ; S. Goldstein, J.L. Lebowitz, R. Tumulka and N. Zanghi, ibid. 96, 050403 (2006).

[4] S. Popescu, A.J. Short and A. Winter, Nature Phys. 2, 754 (2006).

[5] E. J. Heller and S. Tomsovic, Phys. Today 46, 38 (1993).

[6] M. Rigol, V. Dunjko and M. Olshanii, Nature (London) 452, 854 (2008); A. C. Cassidy, C. W. Clark and M. Rigol, Phys. Rev. Lett. 106, 140405 (2011).

[7] M. Rigol and M. Srednicki, Phys. Rev. Lett. 108, 110601 (2012).

[8] F. Reif, Fundamentals of Statistical and Thermal Physics (McGraw-Hill Book Co., New York, 1985).

[9] A. V. Ponomarev, S. Denisov and P. Hanggi, Phys. Rev. Lett. 106, 010405 (2011); A. V. Ponomarev, S. Denisov, P, Hanggi and J. Gemmer, EPL 98, 40011 (2012).

[10] C. Ates, J.P. Garrahan and I. Lesanovsky, Phys. Rev. Lett. 108, 110603 (2012); R. Steinigeweg, A. Khodja, H. Niemeyer, C. Gogolin and J. Gemmer, ibid. 112, 130403 (2014).

[11] V. V. Flambaum and F. M. Izrailev, Phys. Rev. E 56, 5144 (1997).

[12] L. F. Santos, F. Borgonovi and F. M. Izrailev, Phys. Rev.
Lett. 108, 094102 (2012).

[13] M.A. Garcia-March, D.R. Dounas-Frazer and L. D. Carr, Front. Phys. 7, 131 (2012); O. Fialko and D.W. Hallwood, Phys. Rev. Lett. 108, 085303 (2012); O. Fialko, J. Phys. B 47, 045302 (2014).

[14] P.B. Blakie, A.S. Bradley, M.J. Davis, R.J. Ballagh and C.W. Gardiner, Adv. Phys. 57, 363 (2008); A. Polkovnikov, Ann. Phys. 325, 1790 (2010).

[15] M.K. Olsen and A.S. Bradley, Opt. Commun. 282, 3924 (2009).

[16] A. Smerzi, S. Fantoni, S. Giovanazzi and S. R. Shenoy, Phys. Rev. Lett. 79, 4950 (1997); G. J. Milburn, J. Corney, E. M. Wright and D. F. Walls, Phys. Rev. A 55, 4318 (1997); M. Albiez, R. Gati, J. Folling, S. Hunsmann, M. Cristiani and M. K. Oberthaler, Phys. Rev. Lett. 95, 010402 (2005); S. Levy , E. Lahoud, I. Shomroni and J. Steinhauer, Nature (London) 449, 579 (2007).

[17] J. Gillet, M. A. Garcia-March, Th. Busch and F. Sols, Phys. Rev. A 89, 023614 (2014).

[18] T. W.B. Kibble and F. H. Berkshire, Classical Mechanics (Imperial College Press, London, 2012).

[19] A. Altland and F. Haake, New J. Phys. 14, 073011 (2012).

[20] J. Larson, B. M. Anderson and A. Altland, Phys. Rev. A 87, 013624 (2013).

[21] M. Rigol, Phys. Rev. Lett. 112, 170601 (2014). 